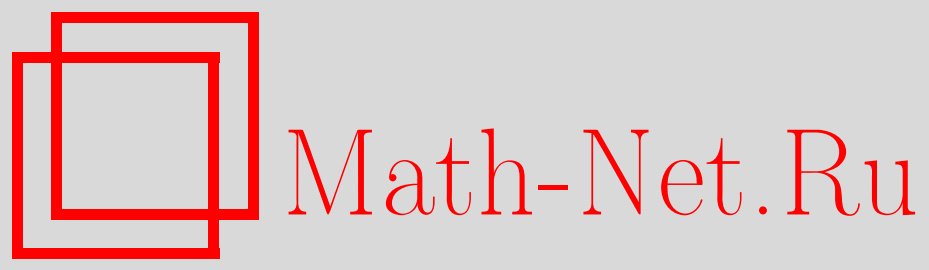

А. В. Карабегов, О деформационном квантовании на кэлеровом многообразии, связанном с квантованием Березина, Функи. анализ и его прил., 1996, том 30, выпуск 2, 87-89

DOI: https://doi.org/10.4213/faa528

Использование Общероссийского математического портала MathNet.Ru подразумевает, что вы прочитали и согласны с пользовательским соглашением http://www . mathnet.ru/rus/agreement

Параметры загрузки:

IP : 54.198 .64 .247

26 апреля 2023 г., 14:55:15

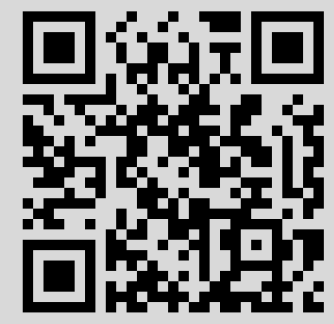




\section{О деформационном квантовании на кэлеровом многообразии, связанном с квантованием Березина}

(C) 1996. А. В. КАРАБЕГОВ

За последние годы появилась серия работ [1-4], в которых изучается деформационное квантование на некоторых классах кэлеровых многообразий, возникающее из квантования Ф. А. Березина [5]. На произвольном симплектическом многообразии деформационное квантование, введенное в [6], может быть построено многими различными способами (см. [7-9]). В настоящей заметке мы приводим конструкцию деформационного квантования на произвольном кэлеровом многообразии, пользуясь подходом Б. В. Федосова [9], и показываем, как такое квантование связано с квантованием Березина. Во всех случаях, рассмотренных в [1-4], деформационное квантование совпадает с построенным в данной работе.

1. Приведем необходимые определения. Деформационным квантованием на симплектическом многообразии $M$ называется структура ассоциативной алгебры в пространстве формальных степенных рядов $\mathcal{F}=C^{\infty}(M)[[\nu]]$, умножение $\star$ в которой задается последовательностью бидифференциальных операторов $\left\{C_{r}(\cdot, \cdot)\right\}, r=0,1, \ldots$, а именно $f \star g=\sum_{r=0}^{\infty} \nu^{r} C_{r}(f, g)$ для $f, g \in \mathcal{F}$. При этом

$$
C_{0}(f, g)=f g, \quad C_{1}(f, g)-C_{1}(g, f)=i\{f, g\},
$$

где $\{\cdot, \cdot\}$ - скобка Пуассона на $M$, очевидным образом распространенная на $\mathcal{F}$.

(Специальное) квантование в смысле $Ф$. А. Березина на $M$ задается семейством ассоциативных алгебр $\mathcal{A}_{\hbar} \subset C^{\infty}(M)$, где параметр $\hbar$ пробегает некоторое множество положительных чисел $E$, имеющее 0 в качестве предельной точки. Затем в прямом произведении $\prod_{\hbar \in E} \mathcal{A}_{\hbar}$ с покомпонентным умножением $*$ выбирается подалгебра $\mathcal{U}$, такая, что для любого элемента $f=f(\hbar) \in \mathcal{U}$, где $f(\hbar) \in \mathcal{A}_{\hbar}$, сушествует предел $\lim _{\hbar \rightarrow 0} f(\hbar)=\varphi(f) \in C^{\infty}(M)$. При этом должен выполняться принцип соответствия: для $f, g \in \mathcal{U}$

$$
\varphi(f * g)=\varphi(f) \varphi(g), \quad \varphi\left(\hbar^{-1}(f * g-g * f)\right)=i\{\varphi(f), \varphi(g)\} .
$$

Нетрудно дополнить определение квантования в смысле Березина так, чтобы связать с ним некоторое деформационное квантование. Предположим, что алгебра $\mathcal{U}$ выбрана таким образом, что ее элементы $f=f(\hbar)$ допускают разложение в асимптотический ряд по $\hbar \rightarrow 0$ с коэффициентами из $C^{\infty}(M), f \sim \sum_{r=0}^{\infty} \hbar^{r} f_{r}$. Определим отображение $\psi: \mathcal{U} \rightarrow \mathcal{F}$, полагая $\psi(f)=\sum_{r=0}^{\infty} \nu^{r} f_{r}$. Мы будем говорить, что квантование Березина ассоциировано с деформационным квантованием (т.е. со структурой алгебры) в $\mathcal{F}$, если $\psi$ - гомоморфизм алгебр. При этом выполнение принципа соответствия (2) будет следовать из (1).

2. Определим теперь деформационное квантование на произвольном кэлеровом многообразии. Пусть $M$ - кэлерово многообразие, $\operatorname{dim}_{\mathbb{R}} M=2 m$, и $\omega$ 
- кэлерова форма на $M$. Для каждой окрестности $U \subset M$ положим $\mathcal{F}(U)=$ $C^{\infty}(U)[[\nu]]$. В $\mathcal{F}(U)$ определено действие формальных рядов дифференциальных операторов. Пусть $U$ - координатная окрестность с координатами $z=$ $\left(z_{1}, \ldots, z_{m}\right)$, в которой определен потенциал $\Phi=\Phi(z, \bar{z})$ кэлеровой формы $\omega$, т.е. $\omega=i \partial \bar{\partial} \Phi$. Обозначим через $\mathcal{L}(U)$ множество формальных рядов дифференциальных операторов, коммутирующих (i) с операторами умножения на антиголоморфные функции $\beta(\bar{z})$ и (ii) с операторами $\partial \Phi / \partial \bar{z}_{k}+\nu \partial / \partial \bar{z}_{k}, k=$ $1, \ldots, m$. Условие (i) означает, что оператор $A \in \mathcal{L}(U)$ можно записать в виде $A=\sum_{r} \nu^{r} a_{r}(z, \bar{z}, \partial / \partial z)$, где $a_{r}$ - дифференциальные операторы. Поэтому $A=A(\bar{z})$ можно считать антиголоморфным сечением тривиального расслоения над $U$, слоем которого является пространство формальных рядов (чисто) голоморфных дифференциальных операторов на $U$. Условие (ii) означает, что $A$ - ковариантно постоянное сечение относительно плоской связности $\nabla=$ $\bar{\partial}+(1 / \nu)[\bar{\partial} \Phi, \cdot]$. Легко видеть, что $\nabla$ зависит только от кэлеровой формы $\omega$. Пользуясь техникой, развитой Б. В. Федосовым в [9], можно доказать, что справедлива

ТЕОРема 1. Для любого элемента $f \in \mathcal{F}(U)$ существует единственньй оператор $A \in \mathcal{L}(U)$ со свободнылм членом $f$, m. е. такой, что $f=A 1$.

Оператор $A$, упомянутый в теореме, обозначим через $L_{f}$. Поскольку операторы из $\mathcal{L}(U)$ образуют алгебру и биективно соответствуют элементам из $\mathcal{F}(U)$, то в $\mathcal{F}(U)$ индуцируется ассоциативное умножение $\star: f \star g=L_{f} L_{g} 1=$ $L_{f} g$ для $f, g \in \mathcal{F}(U)$. Из доказательства теоремы 1 можно также получить, что умножение $\star$ задается бидифференциальными операторами $C_{r}=c_{r}(z, \bar{z}, \partial / \partial \bar{z} \otimes$ $\partial / \partial z), r=0,1, \ldots$, для которых справедливы соотношения (1). Кроме того, операторы $C_{r}$ на всех окрестностях $U$ согласованы и, таким образом, задают деформационное квантование глобально на $M$.

3. Теперь мы свяжем с голоморфным эрмитовым линейным расслоением на кэлеровом многообразии $(M, \omega)$ квантование по Березину на $M$ при условии, что выполнены некоторые технические требования, легко проверяемые на конкретных примерах. Пусть $\pi: L \rightarrow M$ - голоморфное линейное расслоение с эрмитовой метрикой $h$, такое, что кривизна канонической связности на нем равна $i \omega$. Сечению $s \in \Gamma(L)$ отвечает функция $\eta_{s}$ на главном расслоении $L^{\times}=L \backslash\{0\}$, такая, что $\eta_{s}(q) q=s(\pi(q))$ для $q \in L^{\times}$. Она однородна порядка -1 относительно растяжений $q \mapsto t q, t \in \mathbb{C}^{*}$. Метрику $h$ также можно рассматривать как функцию на $L^{\times}$, причем $h(t q)=|t|^{2} h(q)$. Сечения расслоения $L^{n}$ отождествим с однородными порядка $-n$ функциями на $L^{\times}$. Введем на таких функциях эрмитово скалярное произведение. Для функций $\eta_{1}, \eta_{2}$ порядка однородности $-n$ пусть $\tau$ - функция на $M$, такая, что $\tau$ о $\pi=\eta_{1} \bar{\eta}_{2} h^{n}$; тогда положим $\left\langle\eta_{1}, \eta_{2}\right\rangle_{n}=\int \tau \omega^{m} / m$ !. Обозначим через $H_{n}$ гильбертово пространство голоморфных сечений расслоения $L^{n}$ со скалярным произведением $\langle\cdot, \cdot\rangle_{n}$. Для всякого $q \in L^{\times}$в $H_{n}$ существует элемент $e_{q}^{(n)}$ (когерентное состояние), такой, что $\left\langle\eta, e_{q}^{(n)}\right\rangle_{n}=\eta(q)$ для $\eta \in H_{n}$. Расслоение $L$ регулярно в смысле Ронсли, если для всех $n$ функция Ронсли $\epsilon$ на $M$, определяемая равенством $\epsilon(\pi(q))=\left\langle e_{q}^{(n)}, e_{q}^{(n)}\right\rangle_{n} h^{n}(q)$, постоянна. Это условие автоматически выполня- 
ется, если на расслоении $L^{\times}$, транзитивно переставляя слои, действует группа голоморфных автоморфизмов, сохраняющих $h$ (см. [1]). Ковариантным символом оператора $A$ в $H_{n}$ называется функция $\sigma A$ на $M$, определяемая равенством $\sigma A(\pi(q))=\left\langle A e_{q}^{(n)}, e_{q}^{(n)}\right\rangle_{n} /\left\langle e_{q}^{(n)}, e_{q}^{(n)}\right\rangle_{n}$. Пусть $\mathcal{D}$ - алгебра голоморфных однородных порядка 0 относительно растяжений дифференциальных операторов на $L^{\times}$, таких, что ограничения этих операторов на функции порядка однородности - $n$ задают замкнутые операторы в $H_{n}$, содержащие в области определения все когерентные состояния $e_{q}^{(n)}$. Ограничение оператора $A \in \mathcal{D}$ на $H_{n}$ обозначим через $A^{(n)}$. Операторы $A^{(n)}, A \in \mathcal{D}$, биективно соответствуют своим ковариантным символам; поэтому множество $\mathcal{A}_{1 / n}$ всех таких символов является алгеброй относительно умножения, унаследованного из умножения операторов. Мы зададим сейчас алгебру $\mathcal{U}$ на $M$ из п. 1 . Пусть $E=\{1,1 / 2,1 / 3, \ldots\}$. Алгебру $\mathcal{U}$ определим как совокупность элементов $f=f(\hbar) \in \prod_{\hbar \in E} \mathcal{A}_{\hbar}$, таких, что $f(1 / n)=\sum_{k} n^{-d_{k}} \sigma A_{k}^{(n)}$, где $A_{k} \in \mathcal{D}$ - оператор порядка не выше $d_{k}$. Пусть теперь $L$ регулярно в смысле Ронсли, $(\mathcal{F}, \star)$ - деформационное квантование на $M$, введенное в п. 2 , и $\psi$ - отображение, введенное в п. 1.

ТЕорема 2. Отображение $\psi: \mathcal{U} \rightarrow \mathcal{F}$ корректно определено и является гомоморфизмом алгебр.

Доказательство теоремы основано на формуле для символа оператора $A^{(n)}$, $A \in \mathcal{D}: \sigma A^{(n)} \circ \pi=h^{n}\left(A h^{-n}\right)$.

Таким образом, на $M$ возникает квантование Березина, ассоциированное с деформационным квантованием, введенным в п. 2. В работах [1-4] рассматриваются случаи, когда $M$ - обобщенное многообразие флагов или ограниченная симметрическая область. В обоих случаях группа Ли $G$ голоморфных изометрических автоморфизмов многообразия $M$ действует на $L^{\times}$, сохраняя метрику $h$. В качестве $\mathcal{D}$ можно взять алгебру дифференциальных операторов на $L^{\times}$, порожденных инфинитезимальным действием алгебры Ли $\operatorname{Lie}(G)$. Нетрудно показать, что при этом отображение $\psi$ однозначно задает деформационное квантование на $\mathcal{F}$.

Автор пользуется случаем выразить благодарность Б. В. Федосову и Дж. Ронсли, ознакомившим его с препринтами своих работ.

\section{ЛИТЕРАТУРА}

1. Rawnsley J., Cahen M., Gutt S. J. Geom. Phys., 7, 45-62 (1990). 2. Cahen M., Gutt S., Rawnsley J. Trans. Amer. Math. Soc., 337, 73-98 (1993). 3. Cahen M., Gutt S., Rawnsley J. Lett. Math. Phys., 30, 291-305 (1994). 4. Cahen M., Gutt S., Rawnsley J. To appear in Lett. Math. Phys. 5. Березин Ф. А. Изв. АН СССР, сер. матем., 38, 1116-1175 (1974). 6. Bayen F., Flato M., Fronsdal C., Lichnerovich A., Sternheimer D. Ann. Phys., 111, 61-151 (1977). 7. De Wilde M., Lecomte P. Lett. Math. Phys., 7, 487-496 (1983). 8. Omori H., Maeda Y., Yoshioka A. Adv. Math., 85, 224-255 (1991). 9. Fedosov B. V. J. Diff. Geom., 40, 213-238 (1994). 